\title{
Design of High-Isolation Wideband Dual-Polarized Compact MIMO Antennas with Multi-Objective Optimization
}

\author{
Diqun Lu, Lu Wang, Student Member, IEEE, Erfu Yang, and Gang Wang, Member, IEEE
}

\begin{abstract}
Multi-objective optimization design of compact MIMO antennas with canonical structures (viz., transformation or combination by regular shapes) for wideband radiation and fragment structures (viz., combination by discrete fragment patches) for high isolation is proposed and demonstrated. In the design, MOEA/D-DE for optimization of canonical structures and MOEA/D-GO for optimization of fragment structures are combined and iterated. The design is demonstrated with compact bow-tie MIMO antennas sharing a small common ground plane. The four orthogonally deployed bow-tie antennas are optimized by using MOEA/D-DE to acquire dual polarization and wideband radiation. Fragment-type structures on the common ground plane are optimized by using MOEA/D-GO to acquire very high isolation. Both simulation and measurement show that the optimized MIMO bow-tie antennas provide isolation higher than $30 \mathrm{~dB}$ in a relative bandwidth of $40 \%$ (from $2.6 \mathrm{GHz}$ to $3.9 \mathrm{GHz}$ ). With the proposed design technique, dual-band high isolation MIMO antennas may also be designed.
\end{abstract}

Index Terms—antenna; isolation; MIMO; optimization; wideband antennas.

\section{INTRODUCTION}

In recent years, multiple-input and multiple-output (MIMO) technology has become the focus of radars and wireless communications. MIMO radar systems may overcome performance degradations caused by the radar cross section fluctuations, achieve flexible spatial transmit beam design and provide high-resolution spatial spectral estimates [1]. MIMO wireless communication systems may increase channel capacity, enhance data rate and reduce multipath fading [2] [3]. Since strong mutual coupling between antennas will degrade the performance of MIMO systems seriously [4], design of high-isolation MIMO antennas is always desired.

Different structures have been applied to suppress mutual coupling between MIMO antennas. By using parasitic elements [5], neutralization lines [6], decoupling networks [7], coupled resonator decoupling networks [8], defected ground structures [9], and electromagnetic band-gap structures [10], surface current or surface waves were suppressed to lower mutual coupling. These designs

Manuscript received July 29, 2017; revised November 20, 2017. This work was supported in part by National Natural Science Foundation of China under Grant 61671421

D. Lu and L. Wang are with the Department of Electronic Engineering and Information Science, University of Science and Technology of China, Hefei 230027, China.

E. Yang is with the Space Mechatronic Systems Technology Laboratory (SMeSTech), Strathclyde Space Institute, Department of Design, Manufacture and Engineering Management, University of Strathclyde, 75 Montrose Street, Glasgow G1 1XJ, United Kingdom.

G. Wang is with the Key Laboratory of Electromagnetic Space Information, Chinese Academy of Sciences, Hefei 230027, China. (e-mail: gwang01@ustc.edu.cn). achieved isolation around $20 \mathrm{~dB}$ within a relative bandwidth of about $11 \%$ for MIMO antennas. Several other designs challenge higher MIMO isolation. Dummy elements with optimal loads were employed in [11] to generate $25 \mathrm{~dB}$ isolation. Defected wall structures were adopted in [12] to acquire isolation of $35 \mathrm{~dB}$. Fragment-type structures on defected ground were designed to acquired isolation of $36 \mathrm{~dB}$ in [13]. However, the relative bandwidth of $10 \mathrm{~dB}$ return loss in [11]-[13] was below $8 \%$.

Deployment and polarization diversity of MIMO antennas have also been considered to increase the isolation. In [14], radiation in orthogonal directions was arranged to acquire isolation of $22 \mathrm{~dB}$ within a relative bandwidth of $28.6 \%$. The using of polarization diversity of linear-polarized antennas provided an isolation of $16 \mathrm{~dB}$ in a relative bandwidth of $26.5 \%$ in [15], and isolation of $19 \mathrm{~dB}$ in a relative bandwidth of $45.5 \%$ in [16]. In addition to utilization of the polarization diversity, slots on the common ground were reported in [17] to yield an isolation of $15 \mathrm{~dB}$ in a relative bandwidth of $46.8 \%$.

Designing MIMO antennas with higher isolation in a wider bandwidth is still challenging, and can be viewed as a typical multi-objective optimization problem (MOP). Evolution algorithms can be applied to solve MOPs [18]. By using a weighted sum, traditional approaches like different evolution [19] and particle swarm optimizer [20] can combine the objectives into a single function, which yields a single Pareto optimal solution. Recently, Borg multi-objective evolutionary algorithm (Borg MOEA) [21] and multi-objective evolutionary algorithm based on decomposition (MOEA/D) [22] have been reported to solve MOPs, which can optimize multiple objectives simultaneously. Borg MOEA can provide seven mutation and recombination operators for suiting a particular design and uses the $\epsilon$-box dominance archive, while MOEA/D uses the Pareto-based dominance archive, which allows to keep a larger number of points in a given region of the objective space [21]. In addition, by utilizing the information of solution to neighborhood sub-problems and adopting a small neighborhood size, less computation will be cost and the search speed will be improved for MOEA/D [22]. Based on MOEA/D, MOEA/D combined with differential evolution (MOEA/D-DE) can significantly improve the diversity by selecting father-individuals along different search directions [23], and MOEA/D combined with genetic operators (MOEA/D-GO) can converge faster by introducing enhanced genetic operators and work efficiently for design of fragment-type structures [24].

In general, design of compact MIMO antennas includes radiator and isolator structures. For MIMO antennas shown in Fig. 1 including four orthogonally deployed bow-tie antennas, to find a high isolation structure in a very limited circular design space on the shared PCB ground plane, fragment-type structure could benefit. The design includes optimization of radiators using canonical structures and isolators with fragment-type structures. Therefore, an optimization scheme compounding MOEA/D-DE and MOEA/D-GO should work.

This communication is organized as follows. In Section II, geometrical structure of the compact MIMO bow-tie antennas is 
defined. Description of fragment-type isolation structure in a circle area is introduced, which is quite different from that for rectangular design space in [13]. In Section III, general design consideration for multi-objective optimization of MIMO antennas is outlined, and technique for design of high-isolation MIMO bow-tie antennas with rotationally symmetrical fragment-type isolation structure is presented by using compound multi-objective optimization. General performance of broadband bow-tie MIMO antennas is investigated and tested in section IV. Some other design concerns such as efficiency of optimization search and dual-band operation are discussed in section $\mathrm{V}$.

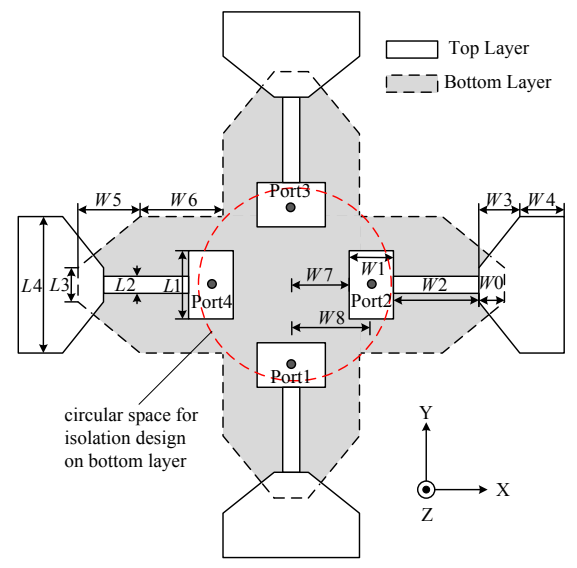

Fig. 1. Architecture of the proposed bow-tie MIMO antennas.

\section{Bow-Tie MiMO ANTENNAS With Fragment-Type IsOlation STRUCTURES}

For the compact bow-tie MIMO antennas shown in Fig. 1, the space for isolation design is a circular area on the common ground plane. Isolation structures with rotational symmetry will benefit the optimization searching greatly.

\section{A. Geometry of Bow-Tie MIMO Antennas}

As shown in Fig. 1, the four bow-tie antennas are deployed along crossed axis with a common area connecting the ground arms of the four bow-tie antennas. Each single bow-tie antenna may have two overlapped arms. The overlapping depth can be adjusted to tune the dual-band or wideband operation. Each bow-tie antenna is fed by a coaxial cable with its inner and outer conductors of the SMA connected to the top and bottom arms, respectively.

Geometrical parameters defining the MIMO bow-tie are shown in Fig. 1. According to the definition, the antenna has an overall dimension of $W=2(W 1+W 2+W 3+W 4+W 7)$ and the overlapping is given by $W 0=W 5+W 6+W 8-(W 1+W 2+W 7)$.

\section{B. Models of Fragment-Type Isolator}

Due to the rotational symmetry of the MIMO bow-tie deployment, fragment cells formatting the fragment-type isolation structure in the circle area will be defined in polar coordinate system. As a result, the design space for isolation can be divided into eight identical parts by the four dashed lines, as shown in Fig. 2(a). Each part is a sector of $45^{\circ}$.

Suppose each $45^{\circ}$ sector in the design space can be divided into fragment sectors. To avoid the use of too small fragment sectors encircling the original point, we may set a large radial step for the sectors encircling the original point, say $6 \mathrm{~mm}$ vs. $2 \mathrm{~mm}$, as shown in Fig. 2(b). By assigning "1" and "0" to the fragment sectors, a design matrix can be constructed. If on the PCB " 1 " is metal (in gray) and " 0 " is air, a $45^{\circ}$ sector with fragment-type structure can be defined.
Different distributions of "1" and "0" define different fragment sectors. Combining the eight $45^{\circ}$ sectors will cover the whole circle design space, as shown in Fig. 2(c). Due to the symmetry of the MIMO antennas, the workload of searching and optimizing the best distribution of fragment sectors can be greatly reduced.

The other way to avoid too small fragment sectors encircling the original point is to leave a blank circular area circling the original point. Fig. 2(c) shows a fragment-type structure in the design space with a circular blank area of radius $R$. As we will demonstrate in section $\mathrm{V}$, the preset circular slot will benefit greatly the searching speed of the fragment-type isolation structures.

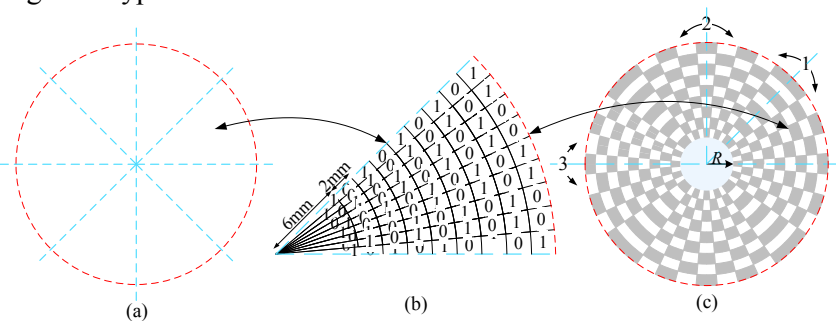

Fig. 2. Models of fragment-type isolation: (a) splitting the circle to eight symmetrical sectors; (b) discretizing the sector and assigning " 0 " or " 1 " to each cell; (c) fragment-type isolation structure.

\section{Design of High-ISOlation Wideband MIMO ANTENNAS}

For the MIMO bow-tie antennas shown in Fig. 1, radiation characteristics, return loss and isolation are usually related with each other. By considering the MIMO antennas as a 4-port network with port 1, 2, 3 and 4 as marked in Fig. 1, basic constrain among the key design indexes can be derived.

With the symmetry of the proposed MIMO antennas, the scattering matrix of the 4-port network can be expressed as

$$
[S]=\left[\begin{array}{llll}
\left|S_{11}\right| e^{j \varphi_{11}} & \left|S_{12}\right| e^{j \varphi_{12}} & \left|S_{13}\right| e^{j \varphi_{13}} & \left|S_{12}\right| e^{j \varphi_{12}} \\
\left|S_{12}\right| e^{j \varphi_{12}} & \left|S_{11}\right| e^{j \varphi_{11}} & \left|S_{12}\right| e^{j \varphi_{12}} & \left|S_{13}\right| e^{j \varphi_{13}} \\
\left|S_{13}\right| e^{j \varphi_{13}} & \left|S_{12}\right| e^{j \varphi_{12}} & \left|S_{11}\right| e^{j \varphi_{11}} & \left|S_{12}\right| e^{j \varphi_{12}} \\
\left|S_{12}\right| e^{j \varphi_{12}} & \left|S_{13}\right| e^{j \varphi_{13}} & \left|S_{12}\right| e^{j \varphi_{12}} & \left|S_{11}\right| e^{j \varphi_{11}}
\end{array}\right],
$$

where $\left|S_{11}\right|$ defines return losses; $\left|S_{12}\right|$ and $\left|S_{13}\right|$ define the isolation between the two neighboring and two opposite antennas, respectively.

For the power fed to one port of the 4-port network, we have the power relation in terms of the scattering parameters

$$
\left|S_{11}\right|^{2}+2 \times\left|S_{12}\right|^{2}+\left|S_{13}\right|^{2}=1-\delta,
$$

where $\delta$ represents losses including radiation of the MIMO array. Higher radiation efficiency usually indicates larger $\delta$.

The incident power $P_{I N}$ and scattered power $P_{S C}$ of the 4-port microwave circuit can be expressed as follows,

$$
\begin{aligned}
P_{I N}-P_{S C} & =\frac{1}{2}[a]^{\dagger}[H][a]>0, \\
{[H] } & =[I]-[S]^{\dagger}[S],
\end{aligned}
$$

where $[a]$ represents the incident power wave of the four ports, $[a]$ represents the complex conjugate transpose matrix of $[a]$, and $[I]$ represents the unit matrix. $[H]$ is a positive definite Hermite quadratic matrix and should satisfy

$$
\operatorname{det}\left[h_{i j}\right]_{k \times k}>0(i, j \leq k \text { and } k=1,2,3,4) \text {, }
$$

where $\left[h_{i j}\right]_{k \times k}$ is the $k$ th-order leading principal minors of $[H]$, and $\operatorname{det}\left[h_{i j}\right]_{k \times k}$ indicates the determinant of $\left[h_{i j}\right]_{k \times k}$. 
From the above equations from (2) to (5), an inequality for the proposed MIMO antennas can be derived as

$$
\left[\left|S_{11}\right|\left|S_{12}\right| \cos \left(\varphi_{11}-\varphi_{12}\right)+\left|S_{12}\right|\left|S_{13}\right| \cos \left(\varphi_{12}-\varphi_{13}\right)\right]^{2}<\frac{\delta^{2}}{4} .
$$

From (6), we can find that the radiation, return loss and isolation are constrained. In addition, the bandwidths for return loss characterized by dynamic range of the first term and for isolation characterized by dynamic range of the second term are also constrained. In general, a larger dynamic range of the first term will result in smaller dynamic range of the second term. Therefore, there is a natural conflict between bandwidths of return loss and isolation.

To achieve satisfactory return loss, high isolation, and other necessary radiation characteristics in a wide bandwidth, multi-objective optimization algorithm should find its applications.

\section{A. Multi-Objective Optimization for the Design}

Typical multi-objective optimization problem for design of MIMO antennas can be expressed as

$$
\begin{aligned}
& \text { minimize } F(X)=\left(f_{1}(X), f_{2}(X), \cdots, f_{n}(X)\right), \\
& \text { subject to } X \in \Omega,
\end{aligned}
$$

where $f_{i}(X)(i=1,2, \cdots, n)$ represent the objectives defining the antenna performance, $X$ is a decision variable which defines the MIMO antenna structure, and $\Omega$ is the design space.

For the 4-port bow-tie MIMO antennas shown in Fig. 1, only two isolation values characterized by $\mathrm{S}_{12}$ and $\mathrm{S}_{13}$, in terms of the scattering parameters, should be concerned due to the symmetry. To ensure high MIMO isolation, typical objective functions can be specified as

$$
\begin{aligned}
& f_{1}(X)=\max \left(Q_{1}-\min _{\omega \in\left[\omega_{1}, \omega_{2}\right]}\left|\left(S_{12}\right)_{d B}\right|, 0\right), \\
& f_{2}(X)=\max \left(Q_{1}-\min _{\omega \in\left[\omega_{1}, \omega_{2}\right]}\left|\left(S_{13}\right)_{d B}\right|, 0\right),
\end{aligned}
$$

where $\left[\omega_{1}, \omega_{2}\right]$ defines the operation band of the MIMO antennas, $Q_{1}$ represents the desired minimum isolation. The objective functions $f_{1}(X)$ and $f_{2}(X)$ guarantee that the minimum value of isolation will be higher than $Q_{1}$ in the desired frequency band.

As for the return loss, objective function can be specified as

$$
f_{3}(X)=\max \left(Q_{2}-\max _{\omega \in\left[\omega_{1}, \omega_{2}\right]}\left|\left(S_{11}\right)_{d B}\right|, 0\right),
$$

where $Q_{2}$ represents the desired minimum return loss in $\mathrm{dB}$ and

\section{$Q_{2}=10$ gives the common requirement}

In general, to ensure the performance of MIMO antennas, other characteristics such as gain, beam width, and radiant efficiency may also be defined. Since the bow-tie antenna has a typical radiation pattern of dipole-likes, here we may just lay stress on the return loss, isolation in a designated bandwidth.

For design of dual-band MIMO antenna, objective functions similar to (8), (9), and (10) can be added to (7) by defining them on the second operation band $\left[\omega_{3}, \omega_{4}\right]$, as will be demonstrated in section $\mathrm{V}$.

\section{B. Flowchart of the Design}

The proposed bow-tie MIMO antennas include both canonical structures defining the radiators and fragment structures defining the isolator. For the isolation design, optimization scheme such as MOEA/D-GO [13] can be used since isolation structure in the design space is defined by distributed fragment sectors. For the return loss control, optimization scheme such as MOEA/D-DE [19] can be used since the antenna is defined by geometric parameters. As a result, the design may be a compound optimization.

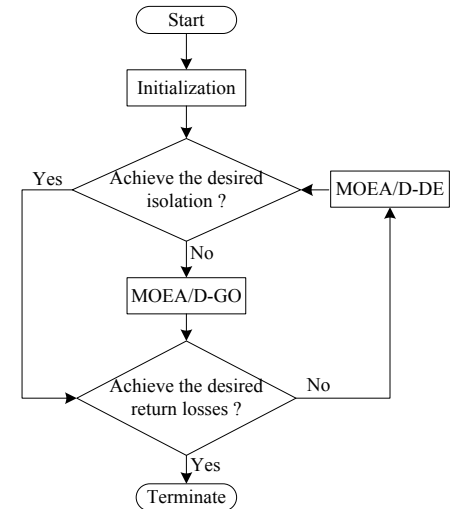

Fig. 3. The design flowchart for the bow-tie MIMO antennas.

Flowchart of the compound optimization is shown in Fig. 3. The optimization design is implemented in the following six steps:

1) Initialization: The initial geometric parameters of the four wideband bow-tie antennas can be obtained by MOEA/D-DE as in [23]. Initial definition of the design space with small fragment sectors as shown in Fig. 2 can be given by attributing the elements of the design matrix either with 1 or 0 randomly.

2) Isolation evaluation and judgment: calculate the values of $S_{12}$ and $\mathrm{S}_{13}$ with EM simulator such as HFSS, and judge if the isolation meets objective functions in (8)-(9).

3) MOEA/D-GO optimization: if not, searching for fragment-type isolation structure with MOEA/D-GO that the MIMO antennas give the desired values of $\mathrm{S}_{12}$ and $\mathrm{S}_{13}$.

4) Return losses evaluation and judgment: calculate the values of $\mathrm{S}_{11}$ of bow-tie MIMO antennas with the optimized fragment-type isolation structure, and judge if the return loss meets (10).

5) MOEA/D-DE optimization: if not, optimizing the bow-tie's geometric parameters with MOEA/D-DE to meet (10)

6) Iteration and termination: For the optimized bow-tie, evaluate the isolation, and then repeat the steps 2) to 5), until both the desired isolation and return loss are achieved.

It should be pointed out that the optimization of bow-tie geometric parameters can also be treated as optimization searching in a discretized "design space" of possible discrete value. As a result, merely MOEA/D-GO can be applied by enlarging the dimension of decision variable $\boldsymbol{X}$ and including all the design objectives defined in (8) to (10).

However, increase of objectives and dimensions of decision variable and design space will remarkably slow down the optimization searching speed of MOEA/D-GO, even if the fastened version MOEA/D-GO-II in [13] is applied. In practice, we may set a maximum iteration number in the codes or we can monitor the isolation and return loss output to decide if there is a need to terminate the searching.

\section{Design of the Bow-Tie MIMO Antenna}

As a demonstration, we design a high-isolation wideband bow-tie MIMO antenna by adopting the fragment-type sectors in Fig. 2 and following the design flowchart in Fig. 3.

Suppose the MIMO antennas are desired to operate in frequency band ranging from $2.6 \mathrm{GHz}$ to $3.7 \mathrm{GHz}$, with isolation higher than $30 \mathrm{~dB}$. The PCB used for the antennas is $\mathrm{F}_{4} \mathrm{~B}$, which has 2-mm thick dielectric substrate of relative dielectric constant of 2.2 and loss tangent of 0.0007 .

After implementing the design flow for objectives defined in (8) to (10) with $\omega_{1}=2.6 \mathrm{GHz}, \omega_{2}=3.7 \mathrm{GHz}, Q_{1}=30$ and $Q_{2}=10$, the design optimization terminates after two iterations.

The optimized bow-tie has geometric parameters listed in Table I. 
The area of circle space for isolation design is $\pi\left(0.22 \lambda_{0}\right)^{2}$, where $\lambda_{0}$ is the wavelength at center frequency of the operation band. The optimized fragment-type isolation structure is shown in Fig. 4 (a). TABLE I

SUGGeSTED DimENSIONS OF WidEBAND BOW-TIE ANTENNAS (IN MILLIMETERS)

\begin{tabular}{cccccccccccc}
\hline \hline$W 1$ & $W 2$ & $W 3$ & $W 4$ & $W 5$ & $W 6$ & $W 7$ & $W 8$ & $L 1$ & $L 2$ & $L 3$ & $L 4$ \\
\hline 7 & 15 & 10 & 14 & 8.3 & 14 & 13 & 17.5 & 12 & 7.5 & 10 & 30 \\
\hline \hline
\end{tabular}

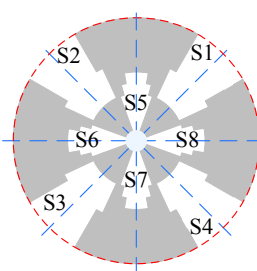

(a)

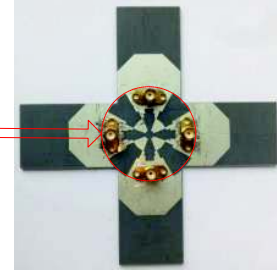

(b)

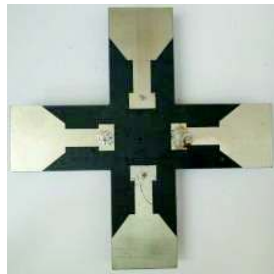

(c)
Fig. 4. (a) layout of the optimized fragment-type isolation structure. (b) Bottom view and (c) top view of the designed bow-tie MIMO antennas.

In the optimization, Pareto front exists for the multi-objective optimization problem. During the optimization, MOEA/D can search the results in uniform directions and obtain a set of Pareto optimal solutions. Fig. 5 depicts the evolution solutions with search generations for MOEA/D-GO and other parameters are fixed in Table I. As shown in Fig. 5, the results of each generation are distributed in different directions and Pareto front can be approached. The Pareto optimal solutions are discretely distributed because the design space is discrete. Once the desired objective is reached, the optimization algorithm will stop. In case the objectives which can't be achieved, the nearest Pareto solution to the desired object can be chosen by monitoring the output results.

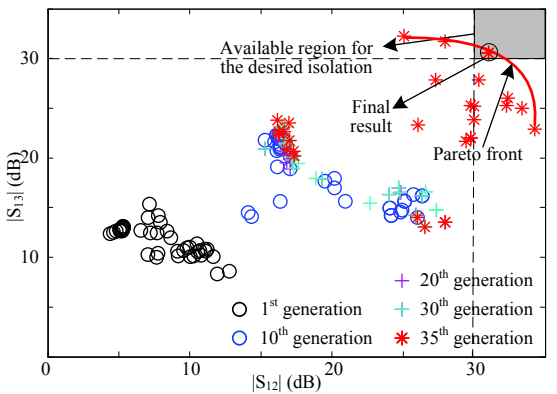

Fig. 5. Simulated solutions with search generations.

\section{Performance of Bow-Tie Mimo Antennas}

For the proposed MIMO antennas and fragment-type isolation structure, prototype of the bow-tie MIMO antennas has been fabricated, as shown in Fig. 4 (b) and (c). We should analyze and demonstrate the MIMO antenna performance.

\section{A. High Isolation in the Wide Bandwidth}

As shown in Fig. 4(a), the optimized fragment-type isolation structure can be viewed as the combination of eight slots, viz., S1 to S8. The lengths of the circumjacent and center slots are $12.2 \approx 0.2 \lambda_{g}$ and $14 \approx 0.21 \lambda_{g}$, respectively, where $\lambda_{g}$ is the guided wavelength at the center frequency of the operation band. The eight slots can be approximately viewed as eight quarter-wavelength slot resonators. When they resonate, the current can't flow between neighboring or opposite antennas.

Fig. 6(a) depicts the current distribution for MIMO antennas with fragment-type isolation at $2.7 \mathrm{GHz}$ when port 1 is excited and other ports are terminated with loads of $50 \Omega$. From Fig. 6 (a), we find that the surface current between neighboring and opposite antennas is trapped by the circumjacent four slots (S1, S2, S3 and S4) and the center four slots (S5, S6, S7 and S8), respectively. Surface current concentrate on the edge of the slots and can't flow to other ports.

Fig. 6 (b) depicts the transmission coefficients $S_{12}$ and $S_{13}$, which characterize the isolation between the two neighboring bow-tie and the two opposite bow-tie, respectively. From Fig. 6 (b), we find that isolation higher than $30 \mathrm{~dB}$ in the desired frequency band ranging from $2.6 \mathrm{GHz}$ to $3.7 \mathrm{GHz}$ can be acquired.

\section{B. MIMO Antenna Performance}

Fig. 7 depicts the simulated and measured S-parameters of the designed bow-tie MIMO antennas. The measured and simulated results are in good agreements. From the measured results in Fig. 7, the return loss is higher than $10 \mathrm{~dB}$ and the isolation is higher than $30 \mathrm{~dB}$ in the operation band from $2.6 \mathrm{GHz}$ to $3.9 \mathrm{GHz}$.

Fig. 8 depicts the simulated and measured radiation patterns of the MIMO antennas, which show a good agreement. The bow-tie antenna attains a gain of $2 \mathrm{~dB}$ at $2.7 \mathrm{GHz}$ and $3.2 \mathrm{~dB}$ at $3.4 \mathrm{GHz}$.

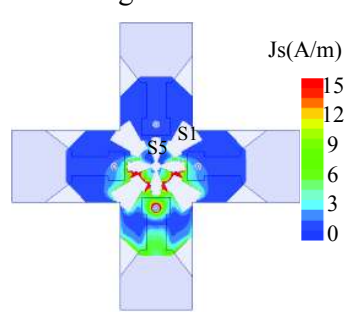

(a)

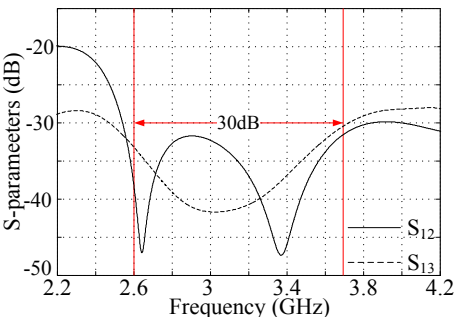

(b)
Fig. 6. Fragment-type isolation with eight slots: (a) simulated current distribution at $2.7 \mathrm{GHz}$ when port 1 is excited; (b) simulated S-parameters.

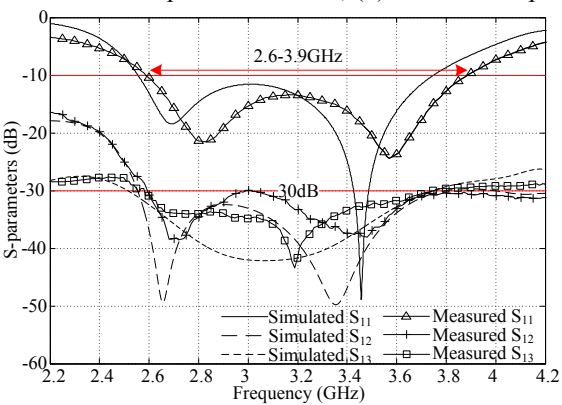

Fig. 7. Simulated and measured reflection coefficients and transmission coefficients of the proposed bow-tie MIMO antennas.

\section{Dual Polarization Operation}

Dual polarization operation is a key feature for this MIMO antenna. Due to orthogonal placement of the bow-tie antennas, dual-polarization characteristics can be achieved, which is beneficial for dual-polarization radars. As shown in Fig. 9(a), when port 1 is excited at $2.7 \mathrm{GHz}$, the current on the top arm mostly flows along the Y-axis. Therefore, when port 2 is excited at $2.7 \mathrm{GHz}$, the current on the top arm flows along the $\mathrm{X}$-axis.

The orthogonal current distribution will yield dual polarization characteristics in the far field, as illustrated in Fig. 9(b). Most of the electric field polarizes along $\mathrm{Y}$-axis when port 1 is excited and along $\mathrm{X}$-axis when port 2 is excited.

The beams of the opposite antennas overlap, which can be used for detecting targets. If antenna 1 is used for transmitting, the same polarization scatter wave can be received by the antenna 3; the antenna 2 and antenna 4 will receive the cross polarization scatter wave. Co-polarization and cross-polarization scatter wave can be obtained by the MIMO array, which is beneficial for detecting targets. 

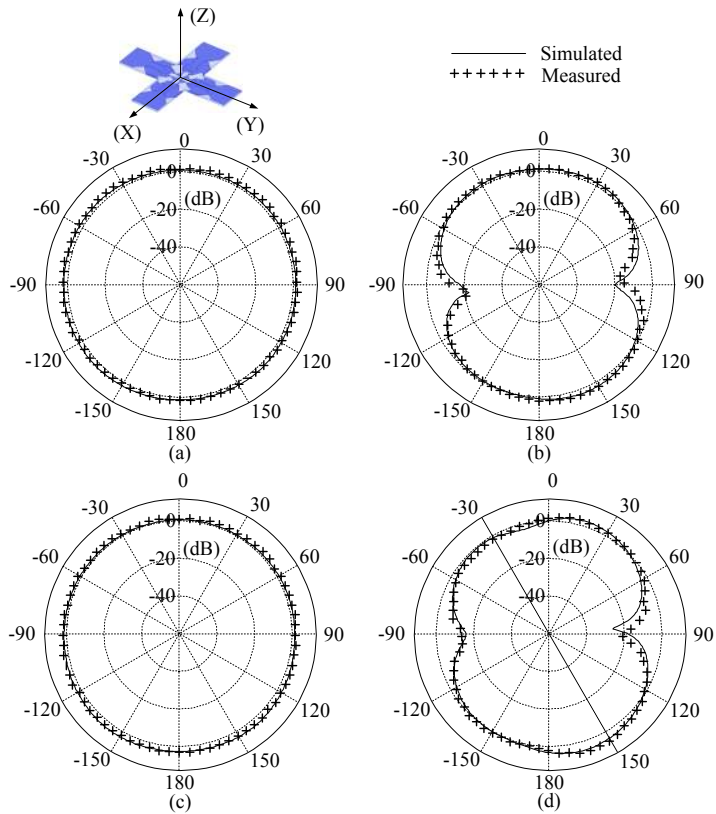

Fig. 8. Simulated and measured radiation patterns when portl is excited. (a) $\mathrm{XOZ}$ plane at $2.7 \mathrm{GHz}$, (b) YOZ plane at $2.7 \mathrm{GHz}$, (c) XOZ plane at $3.4 \mathrm{GHz}$, (d) YOZ plane at $3.4 \mathrm{GHz}$.

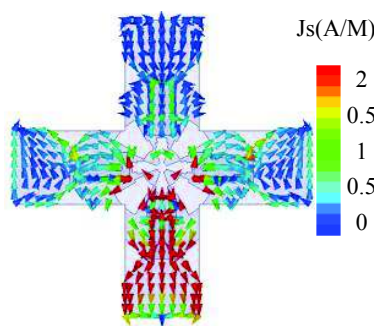

(a)

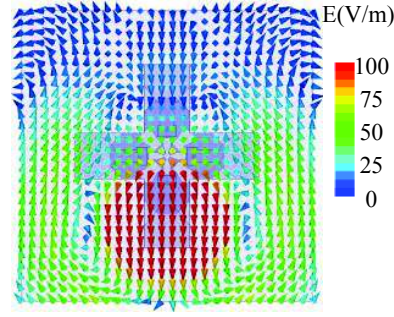

(b)
Fig. 9. Simulated direction of (a) current and (b) E-field when port 1 is excited at $2.7 \mathrm{GHz}$.

TABLE II

COMPARISON BETWEEN PRoposed MIMO ANTENNAS AND PREVIOUSLY PUBLISHED DESIGNS

\begin{tabular}{cccccc}
\hline \hline Ref. & $\mathrm{N}^{*}$ & $\mathrm{~S}^{* *}\left(\lambda_{0}^{2}\right)$ & Relative bandwidth & Isolation & Polarization \\
\hline$[11]$ & 4 & $0.52 \times 0.52$ & $7.7 \%(2.5-2.7 \mathrm{GHz})$ & $\geq 25 \mathrm{~dB}$ & Single \\
{$[14]$} & 4 & $0.7 \times 0.53$ & $28.6 \%(4.5-6 \mathrm{GHz})$ & $\geq 22 \mathrm{~dB}$ & Single \\
{$[15]$} & 3 & $0.93 \times 1.6$ & $25.6 \%(1.7-2.2 \mathrm{GHz})$ & $\geq 16 \mathrm{~dB}$ & Dual \\
{$[16]$} & 4 & $0.88 \times 1.1$ & $45.5 \%(1.7-2.7 \mathrm{GHz})$ & $\geq 19 \mathrm{~dB}$ & Dual \\
{$[17]$} & 4 & $0.55 \times 0.47$ & $46.8 \%(1.8-2.9 \mathrm{GHz})$ & $\geq 15 \mathrm{~dB}$ & Dual \\
$\begin{array}{c}\text { This } \\
\text { paper }\end{array}$ & 4 & $0.6 \times 0.6$ & $40 \%(2.6-3.9 \mathrm{GHz})$ & $\geq \mathbf{3 0 d B}$ & Dual \\
\hline \hline
\end{tabular}

*N means the number of antennas.

**S means the planar area of the single antenna and $\lambda_{0}$ means the wavelength at the center frequency of operation band.

\section{Performance Comparison}

To evaluate the proposed MIMO antennas, characteristics of the proposed bow-tie MIMO antennas and several previously MIMO antennas are listed in Table II.

It is shown in Table II that the bow-tie MIMO antennas with fragment-type isolation provide the highest isolation of $30 \mathrm{~dB}$ in the wide frequency band of $2.6-3.9 \mathrm{GHz}$. Antennas with high isolation are of great importance for MIMO systems.

From Table II, we find that the design in [11] has very narrow bandwidth, although may have pleasant isolation. Wide bandwidths are achieved in [14], [15], [16] and [17]. However, sizes of design in
[15] and [16] are too large, bandwidths of both [14] and [15] are below $30 \%$ and designs in [17] can achieve isolation of only $15 \mathrm{~dB}$. In addition, the bow-tie antenna has a wider bandwidth of $40 \%$ with moderate planar area of $0.6 \lambda_{0} \times 0.6 \lambda_{0} \times 0.02 \lambda_{0}$.

Therefore, the proposed design with a single substrate possesses the highest isolation, dual polarization, and wider bandwidth and provides excellent overall performance.

\section{FURTHER DISCUSSION}

Efficiency of optimization search is a major concern. According to the flowchart in Fig. 3, efficiency of MOEA/D-GO for design of fragment-type isolation structure will seriously affect the efficiency of the MIMO antenna design. By presetting certain specific structures in the design region, the efficiency of MOEA/D-GO for fragment-type isolation structure design can be improved significantly.

In addition, dual-band or multi-band high-isolation MIMO antennas can also be designed with the proposed scheme in Fig. 3. For such designs, the efficiency improvement is of vital concernment.

\section{A. Effect of the Preset Circle Slot}

In the design of the fragment-type isolation structure, a circular slot with radius $R$ is set as shown in Fig. 2(c). It turns out that the preset slot can improve significantly the efficiency of MOEA/D-GO, as shown in Fig. 10. In the comparison of Fig. 10, other parameters of the MIMO array, except for the preset slot radius, are the same and all the designs run on the computer with Intel Core I5-4670@3.4GHz.

It can be observed from Fig. 10 that searching with radius of $2 \mathrm{~mm}$ takes 33 generations (about 14 days) to achieve isolation of $30 \mathrm{~dB}$. As the radius increases from $2 \mathrm{~mm}$ to $6 \mathrm{~mm}$, the searching time decreases from 33 generations (about 14 days) to 8 generations (about 3 day) to acquire the $30 \mathrm{~dB}$ isolation. For radius of $R=8 \mathrm{~mm}, 25 \mathrm{~dB}$ isolation can be acquired after 5 generations (about 2 days). However, a too large radius such as $R=14 \mathrm{~mm}$ will yield very limited isolation because there is small region left for isolation design.

Therefore, by presetting circular slot of moderate radius at the center of the design space, the efficiency of MOEA/D-GO for isolation design can be improved significantly.
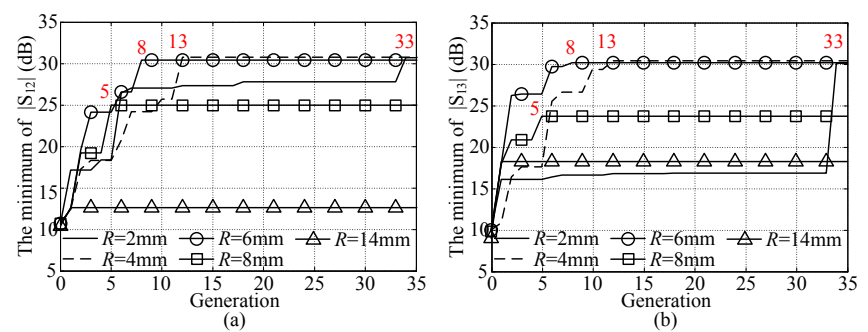

Fig. 10. The minimum isolation of (a) $\mathrm{S}_{12}$ and (b) $\mathrm{S}_{13}$ with search generation for different slot radius $R$.

\section{B. Design of Dual-band MIMO Antennas}

For demonstration, we design a high-isolation dual-band MIMO antenna which operates at $2.5-2.69 \mathrm{GHz}$ and $3.4-3.69 \mathrm{GHz}$ for WiMAX application. For the second operation band, three more objective functions on $\left[\omega_{3}, \omega_{4}\right]$ should be defined as in (8)-(10). After implementing the design flow in Fig. 3 for the optimization objectives with $\omega_{1}=2.5 \mathrm{GHz}, \omega_{2}=2.69 \mathrm{GHz}, \omega_{3}=3.4 \mathrm{GHz}, \omega_{4}=3.69 \mathrm{GHz}$, $Q_{1}=30$ and $Q_{2}=10$, the design optimization terminates after two iterations. The geometric parameters of the optimized bow-tie are listed in Table III and the optimized fragment-type isolation structure is shown in Fig. 11(a).

Fig. 11(b) depicts the return loss and isolation of the designed 
dual-band MIMO bow-tie antennas. From Fig. 11(b), we find that the MIMO array can provide dual-band operations $(2.5-2.75 \mathrm{GHz}$ and $3.35-3.85 \mathrm{GHz}$ ) for WiMAX application. It should be remarked that the isolation in the two operation bands is higher than $30 \mathrm{~dB}$, which is a too high isolation for MIMO wireless communication systems.

TABLE III

SugGeSTED DimENSIONS OF DUAL-BAND BOW-Tie ANTENNAS (IN MILLIMETERS)

\begin{tabular}{cccccccccccc}
\hline \hline$W 1$ & $W 2$ & $W 3$ & $W 4$ & $W 5$ & $W 6$ & $W 7$ & $W 8$ & $L 1$ & $L 2$ & $L 3$ & $L 4$ \\
\hline 7 & 14 & 20 & 4 & 3.8 & 19 & 14 & 17.5 & 13 & 7.7 & 8 & 30 \\
\hline \hline
\end{tabular}

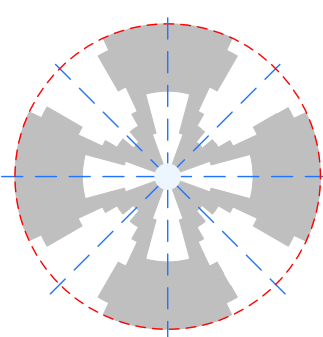

(a)

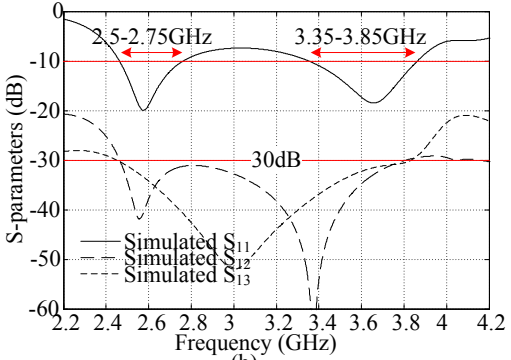

(b)
Fig. 11. (a) Fragment-type isolation structure for dual-band MIMO antennas. (b) Simulated S-parameters of the dual-band MIMO antennas.

\section{CONCLUSION}

By using fragment-type decoupling structures, wideband high-isolation antennas can be designed for MIMO systems. As a result, the MIMO antennas will have both canonical structures for wideband radiation and fragment structures for high isolation. An iterated multi-objective optimization scheme with MOEA/D-DE for optimization of canonical structures and MOEA/D-GO for optimization of fragment structures is proposed for the design.

The proposed design scheme is demonstrated by designing compact MIMO antenna including four orthogonally-deployed bow-tie antennas. Isolation higher than $30 \mathrm{~dB}$ in dual bands or a wideband of relative bandwidth of $40 \%$ has been achieved. Such wideband/dual-band high-isolation MIMO designs are expected to find applications in MIMO radar and MIMO communication systems.

When used in MIMO radar, this combined MIMO antennas may acquire full-polarization scattering data including the V-V, V-H, H-V, and $\mathrm{H}-\mathrm{H}$ in a very convenient manner. Future challenges include the design of high-isolation UWB MIMO antennas for radar applications and the design of compact probe antenna with desired gain and radiation pattern.

\section{References}

[1] L. Z. Xu and J. Li, "Iterative generalized-likelihood ratio test for MIMO radar," IEEE Trans. Signal Process., vol. 55, no. 6, pp. 2375-2385, Jun. 2007. D. Ding and G. Wang, "MOEA/D-GO for fragmented antenna design," Progr. Electromagn. Res. M, vol. 33, pp. 1-15, 2013.

[2] J. W. Wallace, M. A. Jensen, A. L. Swindlehurst and B.D. Jeffs, "Experimental characterization of the MIMO wireless channel: Data acquisition and analysis," IEEE Trans. Wireless Commun., vol. 2, no. 2, pp. 335-343, Mar. 2003.

[3] G. J. Foschini and M. J. Gans, "On limits of wireless communications in a fading environment when using multiple antennas," Wireless Personal Commun., vol. 6, no. 3, pp. 311-335, Mar. 1998.

[4] S. H. Chae, S. K. Oh, and S. O. Park, "Analysis of mutual coupling, correlations, and TARC in WiBro MIMO array antenna," IEEE Antennas Wirel. Propag., vol. 6, pp. 122-125, Apr. 2007.

[5] Z. Li, Z. Du, M. Takahashi, K. Saito, and K. Ito, "Reducing mutual coupling of MIMO antennas with parasitic elements for mobile terminals," IEEE Trans. Antennas Propag., vol. 60, no. 4, pp. 473-481, Feb. 2012.
[6] S. Su, C.-T. Lee, and F.-S. Chang, "Printed MIMO-antenna system using neutralization-line technique for wireless USB-Dongle applications," IEEE Trans. Antennas Propag., vol. 60, no. 2, pp. 456-463, Feb. 2012.

[7] L. Zhao, L. K. Yeung, and K.-L. Wu, "A coupled resonator decoupling network for two-element compact antenna arrays in mobile terminals," IEEE Trans. Antennas Propag., vol. 62, no. 5, pp. 2767-2776, May 2014.

[8] S. N. Venkatasubramanian, L. Li, A. Lehtovuori, et al. "Impact of using resistive elements for wideband isolation improvement," IEEE Trans. Antennas Propag., vol. 65, no. 1, pp. 52-62, Jan. 2017.

[9] C.-Y. Chiu, C.-H. Cheng, R. D. Murch, and C. R. Rowell, "Reduction of mutual coupling between closely-packed antenna elements," IEEE Trans. Antennas Propag., vol. 55, no. 6, pp. 1732-1738, Jun. 2007.

[10] F. Yang and Y. R. Samii, "Microstrip antennas integrated with electromagnetic band-gap EBG structures: A low mutual coupling design for array applications," IEEE Trans. Antennas Propag., vol. 51, no. 10, pp. 2936-2946, Oct. 2003.

[11] L. Zhao and K.-L. Wu, "A decoupling technique for four-element symmetric arrays with reactively loaded dummy elements," IEEE Trans. Antennas Propag., vol. 62, no. 8, pp. 4416-4421, Aug. 2014.

[12] G. Dadashzadeh, A. Dadgarpour, F. Jolani, and B. S. Virdee, "Mutual coupling suppression in closely spaced antennas," IET Microw. Antennas Propag., vol. 5, no. 1, pp. 113-125, Jan. 2011.

[13] D. Ding, G. Wang, and L. Wang, "High-efficiency scheme and optimization technique for design of fragment-type isolation structure between multiple-input and multiple-output antennas," IET Microw. Antennas Propag., vol. 9, no. 9, pp. 933-939, Jun. 2015.

[14] H.-T. Hu, F.-C. Chen and Q.-X. Chu, "A compact directional slot antenna and its application in MIMO array," IEEE Trans. Antennas Propag., vol. 64, no. 12, pp. 5513-5517, Dec. 2016.

[15] Y. Wang and Z. Du, "Dual-polarized slot-coupled microstrip antenna array with stable active element pattern," IEEE Trans. Antennas Propag., vol. 63, no. 9, pp. 4239-4244, Sep. 2015.

[16] J.-S. Row and Y. H. Chen, "Wideband planar array with broad beamwidth and low cross polarization," IEEE Trans. Antennas Propag., vol. 63, no. 9, pp. 4161-4165, Sep. 2015.

[17] A. MoradiKordalivand, T. A. Rahman, and M. Khalily, "Common elements wideband MIMO antenna system for WiFi/LTE accesspoint applications," IEEE Antennas Wireless Propag. Lett., vol. 13, pp. 1601-1604, Aug. 2014.

[18] P. Rocca, M. Benedetti, M. Donelli, D. Franceschini, and A. Massa, "Evolutionary optimization as applied to inverse scattering problems," Inverse Problems, vol. 25, no. 12, pp. 1-41, Dec. 2009

[19] P. Rocca, G. Oliveri, and A. Massa, "Differential evolution as applied to electromagnetics," IEEE Antennas Propag. Mag., vol. 53, no. 1, pp. 38-49, Feb. 2011.

[20] T. Moriyama, F. Viani, M. Salucci, F. Robol, and E. Giarola, "Planar multiband antenna for $3 \mathrm{G} / 4 \mathrm{G}$ advanced wireless services," IEICE Electron. Express, vol. 11, no. 17, pp. 1-10, Sep. 2014.

[21] J. Nagar and D. H. Werner, "A comparison of three uniquely different state of the art and two classical multiobjective optimization algorithms as applied to electromagnetics," IEEE Trans. Antennas Propag., vol. 65, no. 3, pp. 1267-1279, Mar. 2017.

[22] H. Li and Q. Zhang, "MOEA/D: amultiobjective evolutionary algorithm based on decomposition," IEEE Trans. Evol. Comput., vol. 11, no. 6, pp. 712-731, Dec. 2007.

[23] D. Ding and G. Wang, "Modified multiobjective evolutionary algorithm based on decomposition for antenna design," IEEE Trans. Antennas Propag., vol. 61, no. 10, pp. 5301-5307, Oct. 2013.

[24] D. Ding and G. Wang, "MOEA/D-GO for fragmented antenna design," Progr. Electromagn. Res. M, vol. 33, pp. 1-15, 2013. 